\title{
Cryptococcemia According to Immune Status: An Analysis of 65 Critical Cases
}

\author{
Ruochan Chen · Yiya Zhang · Pengcheng Zhou · Xiao Zhong · \\ Mingxiang Zou • Yanming Li · Yan Huang (D)
}

Received: September 28, 2020 / Accepted: November 19, 2020 / Published online: December 16, 2020

(C) The Author(s) 2020

\section{ABSTRACT}

Introduction: Cryptococcemia is associated with poor prognosis among patients with cryptococcosis. However, there are limited data on the clinical features of cryptococcemia, particularly among patients with different immune

Ruochan Chen and Yiya Zhang contributed equally to this work.

R. Chen · X. Zhong · Y. Huang ( $\varangle)$

Department of Infectious Disease, Xiangya Hospital, Central South University, Changsha, Hunan, China e-mail: drhyan@126.com

R. Chen · Y. Zhang · P. Zhou · X. Zhong · Y. Huang Hunan Key Laboratory of Viral Hepatitis, Xiangya Hospital, Central South University, Changsha, Hunan, China

Y. Zhang

Department of Dermatology, Xiangya Hospital, Central South University, Changsha, Hunan, China

Y. Zhang

National Clinical Research Center for Geriatric Disorders, Xiangya Hospital, Central South University, Changsha, Hunan, China

P. Zhou

Department of Infection Control Center, Xiangya Hospital, Central South University, Changsha, Hunan, China

M. Zou · Y. Li

Department of Clinical Laboratory Center, Xiangya Hospital, Central South University, Changsha, China statuses. This study assessed the largest number of cases diagnosed with cryptococcemia, to the best of our knowledge.

Methods: Demographic and clinical data of patients with positive blood culture results for Cryptococcus were obtained from medical records at the Xiangya Hospital (2010-2019).

Results: A total of 65 patients were diagnosed and treated for cryptococcemia, of which 53 (82\%) immunosuppressed patients were afflicted with HIV $(12 \%, 8 / 65)$, tuberculosis $(8 \%$, $5 / 26)$, liver cirrhosis $(6 \%, 3 / 65)$, chronic renal failure $(6 \%, 3 / 65)$, nephrotic syndrome $(13 \%$, $7 / 65)$, systemic lupus erythematosus $(8 \%, 5 / 65)$, chronic glomerulonephritis (11\%, 6/65), malignant diseases $(19 \%, 10 / 65)$, and diabetes $(11 \%, 6 / 65)$. Most patients $(85 \%, 55 / 65)$ presented with fever. Other symptoms, such as cough, headache, enlarged lymph nodes, liver, or spleen, and septic shock, were also reported. Typically, the sites of infection included the central nervous system, lung, skin, bone, abdomen, endometrium, lymph node, and blood. Although early systemic antifungal therapy was administered to 61 patients within $48 \mathrm{~h}$ of hospitalization, the 60-day mortality rate was higher in the immunosuppressed group $(53 \%)$ than in the immunocompetent group $(8 \%)$.

Conclusions: Our study indicated that patients with different immune statuses presented different clinical features. Immunosuppressed patients with cryptococcemia presented a 
higher risk of mortality with poor prognosis, which required intense attention and treatment in time.

Keywords: Clinical features; Clinical outcome; Cryptococcemia; Immune status; Immunosuppression

\section{Key Summary Points}

\section{Why carry out this study?}

There are limited data on the clinical features of cryptococcemia, particularly among patients with different immune statuses.

This study assessed the largest number of cases diagnosed with cryptococcemia, to the best of our knowledge.

\section{What was learned from the study?}

Our study indicated that patients with different immune statuses presented different clinical features, and reported several unusual routes of infection.

Immunosuppressed patients with cryptococcemia presented a higher risk of mortality with poor prognosis, which required intense attention and treatment in time.

\section{DIGITAL FEATURES}

This article is published with digital features, including a summary slide, to facilitate understanding of the article. To view digital features for this article go to https://doi.org/10.6084/ m9.figshare.13259267.

\section{INTRODUCTION}

Cryptococcosis is an opportunistic infection caused by Cryptococcus neoformans which occurs in severely immunosuppressed patients [1]. The infection spreads throughout the human body, in particular, the central nervous system (CNS) [2]. Typically, C. neoformans is isolated from $10-30 \%$ of patients afflicted with cryptococcemia or disseminated cryptococcosis [3]. Unlike other clinical subtypes of cryptococcosis, cryptococcemia is an indicator of poor prognosis among patients with cryptococcal infection of the CNS [4].

In the recent decade, the incidence of cases involving organ transplantation, malignant diseases, administration of corticosteroids, and other immune inhibitors has increased. Such patients show an immunocompromised status and are susceptible to cryptococcosis with a high mortality rate; however, only two clinical cases of cryptococcemia have been reported in the literature $[5,6]$. In the present retrospective cohort study, we investigated the implications of cryptococcemia to understand its clinical significance.

\section{METHODS}

\section{Patients}

C. neoformans was isolated from the blood culture of patients (January 2010 to December 2019) at the Xiangya Hospital of Central South University (Changsha, Hunan, China). The inclusion criteria was positive blood culture of C. neoformans. The hospital is a tertiary medical center with a capacity of 3500 beds that provides both primary and tertiary referral care, and includes a bone marrow and solid organ transplantation center. Before 2015, the isolated strains of $C$. neoformans were cultured and identified in our hospital using India ink stain. After 2015, the fungi strain was identified by mass spectroscopy. Serum cryptococcal antigen was not detected in our hospital. The automatic fungal BD BACTEC ${ }^{\mathrm{TM}}$ Instrumented Blood Culture System (Becton Dickinson Diagnostic Instrument Systems, Sparks, MD, USA) was applied to facilitate rapid reporting. All blood samples obtained from patients with suspected fungal infection were incubated routinely for 15 days before 'no growth' was reported. The list of patients among whom C. neoformans had 
been isolated from the blood was obtained from the computer database of the clinical microorganism isolation system. The study protocol was approved by the Ethics Committee of Xiangya Hospital (no. 2018091080). Data of the patients submitted were anonymized.

\section{Data}

The medical records of the patients were obtained from the database of Xiangya Hospital and reviewed carefully. We also collected data regarding demographics and clinical manifestations, such as age, sex, duration of hospitalization, predisposing conditions, significant symptoms, physical and laboratory findings, antifungal treatment, and clinical outcomes.

\section{Definitions}

The diagnosis of cryptococcemia was confirmed by two specialists based on the following criteria: (1) the patients demonstrated symptoms and signs of infection, and (2) their blood culture yielded positive results of infection at least once. Accordingly, the patients were divided into two subgroups (the immunosuppressed and immunocompetent groups). As per the definition of pneumonia (ventilator-associated pneumonia and non-ventilator-associated pneumonia) put out by the United States Centers for Disease Control, immunosuppressed patients represented those (1) with neutropenia, i.e., an absolute neutrophil count or total white blood cell count $(\mathrm{WBC})<500 / \mathrm{mm}^{3}$, (2) with leukemia, lymphoma, or acquired immune deficiency syndrome (AIDS) with CD4 count < 200 cells $/ \mathrm{mm}^{3}$, (3) who underwent splenectomy, (4) with a history of solid organ or hematopoietic stem cell transplant, (5) who underwent cytotoxic chemotherapy, and (6) who took steroids (excluding inhaled steroids) daily for $>2$ weeks. The immunocompetent group included subjects with no underlying diseases or who had not been properly diagnosed with the disease. Sepsis and septic shock were defined according to the criteria of The Third International Consensus Definitions for Sepsis and Septic Shock (Sepsis-3) [7]. The clinical outcomes of the patients were considered after they were admitted to the hospital (day 1). Signs of improvement, deterioration, and cause of death were recorded until their 60th day of hospitalization. Improvement was assessed in terms of a favorable clinical response to therapy at the end of hospitalization. Antifungal treatment was analyzed using a onedose-received approach (the initial dose and treatment after admission), and empirical treatment was evaluated based on the antifungal treatment administered before positive culture results were obtained (except for antifungal prophylaxis). According to European guidelines for primary antifungal prophylaxis in adult hematology patients, azoles are considered the first choice for primary antifungal prophylaxis for patients receiving intensive remission induction chemotherapy for AML or MDS. Patients with lymphoma tend to be at low risk of IFD. Therefore, no recommended antifungal protocol was mentioned in this guideline [8].

\section{Statistical Analysis}

Differences in qualitative variables between the two groups were analyzed using the chi-squared test with continuity correction or Fisher's exact test, as appropriate. Quantitative data were compared using the $t$ test. Multivariate analysis was not performed as the patient pool was not large enough to yield reliable results. All statistical analyses were performed using SPSS v.19 (IBM, Armonk, NY, USA). Analysis items with $p<0.05$ were considered statistically significant.

\section{RESULTS}

\section{Demographics}

In total, 65 patients with cryptococcemia were diagnosed at Xiangya Hospital from January 2010 through December 2019. Eighty-two percent of the patients were reported to have underlying immunosuppressive conditions; the number of patients in this group was 4.5 times higher than that of those in the 
immunocompetent group. No significant differences in age, gender, or the duration of hospitalization were observed between the two groups (Table 1).

\section{Underlying Diseases}

Twelve patients with cryptococcemia did not show evidence of underlying immune suppression. Among the remaining 53 immunosuppressed patients, treatment with immunosuppressive drugs $(n=21)$, malignant diseases $(n=10)$, and AIDS $(n=8)$ were the three major predisposing conditions. The patients with chronic renal failure, nephritic syndrome, systemic lupus erythematosus, and chronic glomerulonephritis underwent treatment comprising immunosuppressive drugs, such as glucocorticoids and mycophenolate. Four patients had lymphoma and one was diagnosed with glioblastoma. Six subjects were receiving chemotherapy or radiotherapy prior to being diagnosed with cryptococcemia; however, none of the patients who underwent immunosuppressive therapy was a recipient of bone marrow or solid organ transplant in our study. Nevertheless, the distributions of other predisposing conditions, such as tuberculosis, liver cirrhosis, and diabetes, are shown in Table 2.

\section{Clinical Manifestations}

Fifty-six of the 65 patients admitted reported fever (axillary temperature $\geq 37^{\circ} \mathrm{C}$ ). Thirtyeight of the 56 patients presented with high
Table 2 The underlying diseases of 65 patients with cryptococcaemia

\begin{tabular}{ll}
\hline Underlying diseases & $\boldsymbol{n}$ (\%) \\
\hline AIDS & $8(11.54 \%)$ \\
Tuberculosis & $5(7.69 \%)$ \\
Liver cirrhosis & $3(5.66 \%)$ \\
CRF & $3(5.66 \%)$ \\
NS & $7(13.20 \%)$ \\
SLE & $5(9.43 \%)$ \\
Chronic glomerulonephritis & $6(11.32 \%)$ \\
Malignant diseases & $10(18.87 \%)$ \\
DM & $6(11.32 \%)$ \\
No underlying diseases & \\
\hline
\end{tabular}

AIDS Acquired immune deficiency syndrome, $C R F$ chronic renal failure, NS nephrotic syndrome, SLE systemic lupus erythematosus, $D M$ diabetes mellitus

${ }^{a}$ Cases with clear description of no underlying diseases or failure to identify such diseases

fever (axillary temperature $\geq 39^{\circ} \mathrm{C}$ ). In the immunosuppressed group, 47 patients $(88 \%$, $47 / 53$ ) had fever; however, only 20 patients $(38 \%, 20 / 53)$ were critically ill. Sixteen immunosuppressed patients $(29 \%, 16 / 53)$ and 8 immunocompetent patients $(67 \%, 8 / 12)$ had cryptococcal meningitis, which was confirmed via cerebrospinal fluid culture.

Immunosuppressed patients were more likely to have septic shock at admission than immunocompetent patients $(47 \%$ vs. $0 \%$,

Table 1 The basal information of 65 patients with cryptococcaemia under different immunological status

\begin{tabular}{llll}
\hline & Immunosuppressed $(\boldsymbol{n}=\mathbf{5 3})$ & Immunocompetent $(\boldsymbol{n}=\mathbf{1 2})$ & $\boldsymbol{P}$ value \\
\hline Demographic features & & & \\
Male/female ratio & $33 / 9$ & $7 / 5$ & 0.873 \\
Age (years) & $48.0 \pm 18.1$ & $54.2 \pm 15.4$ & 0.334 \\
Length of hospital stay (days) & $18(5,92)^{\mathrm{a}}$ & $50(18,100)^{\mathrm{a}}$ & 0.089 \\
\hline
\end{tabular}

${ }^{a}$ Numbers are median plus ranges 
$p=0.003)$. Several patients had abnormal laboratory results, such as those regarding WBC count, procalcitonin, alanine transaminase, and blood urea nitrogen levels. The clinical manifestations are summarized in Table 3.

\section{Organ Involvement Among 65 Patients with Cryptococcemia}

In our study, most cases of cryptococcemia involved infections of the CNS (55\%, 36/65) and lungs $(23 \%, 15 / 65)$, and disseminated infection (45\%). Positive results of cerebrospinal fluid culture or India ink stain suggested meningeal involvement. Pulmonary cryptococcosis was confirmed by the fungal culture of endotracheal aspirates, bronchoalveolar lavage, or pleural fluid. Cutaneous cryptococcosis was diagnosed based on skin tissue biopsy. One patient with pre-existing liver cirrhosis developed cryptococcal peritonitis proven on culture, whereas another female patient had $C$. neoformans isolated from a culture of endometrial tissue. Twenty-nine patients (45\%) had disseminated infections and C. neoformans affected two or more organs (Table 4). There was a higher incidence of disseminated infection among immunosuppressed patients than among immunocompetent patients.

\section{Antifungal Therapy}

Two of four immunosuppressed patients (6\%, 4/65) had AIDS, one had lymphoma, and the other had liver cirrhosis. These patients did not receive prior antifungal therapy and died before their blood cultures could be obtained. The remaining 61 patients were treated with early systemic antifungal therapy, which was initiated within $48 \mathrm{~h}$ of hospitalization. Thirty patients $(46 \%, 30 / 65)$ received amphotericin B (AmB) alone or followed by sequential treatment with other antifungal drugs. Ten patients $(15 \%, 10 / 65)$ were treated with AmB in combination with 5-fluorocytosine (5-FC) or fluconazole (FCZ). Three patients received oral FCZ and

Table 3 Clinical manifestations of 65 patients with cryptococcaemia

\begin{tabular}{llll}
\hline & Immunosuppressed $(\boldsymbol{n}=\mathbf{5 3})$ & Immunocompetent $(\boldsymbol{n}=\mathbf{1 2})$ & $\boldsymbol{P}$ \\
\hline Fever & $47(88.30 \%)$ & $9(75.00 \%)$ & 0.155 \\
$\mathrm{~T} \geq 39{ }^{\circ} \mathrm{C}$ & $20(37.73 \%)$ & $10(83.33 \%)$ & 0.009 \\
Symptoms/signs of meningitis & $16(29.41 \%)$ & $8(66.66 \%)$ & 0.067 \\
Cough & $7(13.20 \%)$ & $1(0.83 \%)$ & 0.006 \\
Septic shock & $25(47.06 \%)$ & $0(0 \%)$ & 0.012 \\
Enlarged lymph nodes & $3(17.65 \%)$ & $7(77.78 \%)$ & 0.003 \\
Enlarged liver/spleen & $3(5.88 \%)$ & $6(55.56 \%)$ & 0.004 \\
Abnormal chest x-ray/CT & $9(16.98 \%)$ & $1(8.33 \%)$ & 0.143 \\
Laboratory results & & & \\
WBC/N (elevated) & $22(41.18 \%)$ & $9(77.78 \%)$ & 0.025 \\
PCT (elevated) & $16(29.41 \%)$ & $8(66.67 \%)$ & 0.017 \\
ALT (elevated) & $6(11.76 \%)$ & $5(44.44 \%)$ & 0.014 \\
BUN (elevated) & $19(35.29 \%)$ & $1(8.33 \%)$ & 0.036 \\
\hline
\end{tabular}

$T$ temperature, $C T$ computed tomography, $W B C$ white blood cell, $N$ neutrophils, $P C T$ procalcitonin, $A L T$ alanine aminotransferase, $B U N$ blood urea nitrogen 
Table 4 Sites of infections from 65 patients with cryptococcaemia

\begin{tabular}{ll}
\hline Site of infection & $\boldsymbol{n}(\%)$ \\
\hline CNS & $36(55.38 \%)$ \\
Lung & $15(23.07 \%)$ \\
Skin & $10(15.38 \%)$ \\
Bone & $8(12.30 \%)$ \\
Abdominal infection & $3(3.85 \%)$ \\
Endometrial infection & $3(3.85 \%)$ \\
Lymph node & $10(15.38 \%)$ \\
Disseminated infection ${ }^{a}$ & $29(44.61 \%)$ \\
\hline a Infection of more than two organs
\end{tabular}

three were treated empirically with caspofungin alone, as the dosage could not be changed due to their severely impaired liver and renal functions (Table 5).

\section{Clinical Outcomes}

The clinical outcomes of all 65 patients were recorded on the 60th day after admission. In the immunosuppressed group, there were 28 cases

Table 5 Antifungal therapy of 65 patients with cryptococcaemia

\begin{tabular}{lc}
\hline Treatment & $n$ \\
\hline $\mathrm{AmB}^{\mathrm{a}}$ & $30(46.15 \%)$ \\
$\mathrm{FCZ}$ & $3(3.85 \%)$ \\
$\mathrm{AmB}+5-\mathrm{FC}$ & $10(15.38 \%)$ \\
$\mathrm{AmB}+\mathrm{FCZ}$ & $10(15.38 \%)$ \\
$\mathrm{AmB}+5 \mathrm{FC}+\mathrm{VCZ}$ & $3(3.85 \%)$ \\
$\mathrm{Caspofungin}$ & $3(3.85 \%)$ \\
$\mathrm{No}$ Treatment & $4(6.15 \%)$ \\
\hline $\begin{array}{l}5-F C \text { 5-fluorocytosine } \\
\text { anclude } 7 \text { patients (10.76\%, } n=65)\end{array}$ \\
$\begin{array}{l}\text { amphotericin B (AmB) alone and other patients treated } \\
\text { with the initiated AmB treatment followed by other } \\
\text { antifungal drugs sequentially, such as fluconazole }(F C Z) \\
\text { and voriconazole }(V C Z)\end{array}$
\end{tabular}

of death $(53 \%, 28 / 53), 3$ cases of deterioration (unfortunately, these patients died soon after admission due to refractory hypotension and multiple organ failure), and 22 cases of clinical improvement $(41 \%, 22 / 53)$. The rate of acute mortality (within 14 days after admission) was high among patients in the immunosuppressed group $(51 \%, 27 / 53)$. The rates of mortality, improvement, and deterioration were 53\%, $41 \%$, and $6 \%$, respectively. The only patient who died in the immunocompetent group was an elderly patient with disseminated cryptococcosis. That patient died soon after admission on account of multiple organ failure. There were significant differences in the rates of mortality and clinical improvement between the two groups of patients with different immune statuses $(p<0.05)$ (Table 6$)$.

\section{DISCUSSION}

C. neoformans enters the respiratory tract, gastrointestinal system, and damaged skin and mucosa of the human body, leading to opportunistic infection of the CNS, lung, skin, bone, blood, and other organs [9]. The incidence and mortality rates among patients with cryptococcemia are increasing; however, there are limited data regarding the clinical significance of cryptococcemia. Our study, involving 65 patients, revealed that most cases of cryptococcemia occur among immunosuppressed patients; however, 12 immunocompetent patients were also diagnosed with cryptococcemia. During the past decade, the number of reported cases involving Cryptococcus has been on the rise due to immunosuppression caused by predisposing factors, such as AIDS, organ transplantation, long-term use of corticosteroid, and other immunosuppressive agents, as well as malignancies $[10,11]$. We reported several underlying diseases that are uncommon among patients with cryptococcosis, such as tuberculosis and diabetes. However, other underlying factors among immunocompetent patients remain to be discovered.

The clinical symptoms of cryptococcemia are generally nonspecific, which therefore results in a wrong diagnosis or no diagnosis. In our study, 
Table 6 The 60-day prognosis of 65 patients with cryptococcaemia

\begin{tabular}{|c|c|c|c|c|}
\hline & \multicolumn{2}{|c|}{ Failure of treatment } & \multirow[t]{2}{*}{ Improvement } & \multirow[t]{2}{*}{$P$} \\
\hline & Mortality & Deterioration & & \\
\hline Immunosuppressed & $28(52.94 \%)$ & $3(5.88 \%)$ & $22(41.18 \%)$ & 0.003 \\
\hline Immunocompetent & $1(8.33 \%)$ & $1(08.33 \%)$ & $9(77.78 \%)$ & \\
\hline
\end{tabular}

the most frequent symptom of cryptococcemia was fever ( $88 \%$ of immunosuppressed patients, and $75 \%$ of immunocompetent patients presented with fever on admission). Eighty-three percent of the admitted cases in the immunocompetent group presented with high fever as opposed to $38 \%$ in the immunosuppressed group. Among immunosuppressed patients, $47 \%$ developed septic shock as opposed to none in the immunocompetent group. Patients with cryptococcemia showed a high incidence of CNS and lung infections [12]. In our study, 45\% of patients had disseminated cryptococcosis, which included infections of the CNS, lungs, abdominal cavity, skin, bone, endometrium, and lymph nodes. A case of peritonitis occurred in a patient with underlying liver cirrhosis, which was identified as a risk factor for cryptococcal peritonitis [13]. These findings suggested that microorganism cultures should be obtained from multiple sites, including the cerebrospinal fluid, sputum, bronchoalveolar lavage fluid, pleural fluid, ascitic fluid, blood, and specimens from other parts of the patient's body suspected to be infected with $C$. neoformans (for example, patients with fever of unknown origin or at high risk).

In our study, the WBC counts among immunocompetent patients were high due to their satisfactory immune response. In accordance with studies from other groups, three patients also presented with elevated eosinophil granulocyte counts, all of whom were in the immunocompetent group [14]. This could be due to the better immune response among immunocompetent patients. We should pay attention to the differences in laboratory results between the immunosuppressed and immunocompetent groups.
Patients with AIDS or under immunosuppressive therapy are at increased risk for development of cryptococcal diseases. It is worth noting that, in our study, tuberculosis (TB) infection was the predisposing condition in up to $9 \%(5 / 53)$ of the patients when cryptococcaemia developed. TB infection has been closely associated with the immunity condition of the human body [15]. TB disease suggests the impaired immune function of human, and mycobacterium tuberculosis (Mtb) can also cause immune responses evasion and disrupt the crosstalk between innate immunity and adaptive immunity $[16,17]$. The impaired host defense is likely to predispose patients with TB infection to the invasion of C. neoformans from the respiratory or gastrointestinal tract into bloodstream. As Mtb infection remains a global public health problem in an era of increasing antibiotic resistance, we should pay more attention to the association between TB and cryptococcus.

The current guidelines do not focus specifically on cryptococcemia. According to the Clinical Practice Guidelines for the Management of Cryptococcal Disease published in 2010, the treatment for cryptococcemia or disseminated cryptococcosis is the same as that for cryptococcosis of the CNS [18]. However, cryptococcemia is a complicated disease with various underlying conditions, and, hence, requires comprehensive therapy. From our perspective, cryptococcemia is serious clinical conditions with possible multi-organ involvement. We should pay attention to the prevention of acute stress ulcers, and the maintenance of important organ functions, like heart, brain, liver, and kidney. We should also tailor the individual therapy to each patient based on microbiological results, such as burden of exposure and/or 
virulence factors of the infecting strain, and clinical resolution. The specific therapeutical program should be adjusted regarding the immune status of patients, as the clinical characteristics were different according to our research. Further studies and systemic research are necessary to determine the optimal therapeutic regimen for patients with cryptococcemia.

Moreover, the patients with cryptococcemia presented a high acute mortality rate, particularly among those with immunosuppression. The 14-day mortality rate was $51 \%$, which is consistent with the results of a previous study [6]. Furthermore, $6 \%$ of the patients succumbed to cryptococcemia before blood culture results could be evaluated. Patients who received antifungal therapy within $48 \mathrm{~h}$ survived. These findings indicated that cryptococcemia represents an acute, fulminant form of cryptococcosis, which demands early diagnosis and prompt antifungal therapy.

There are several limitations of our study. First, we did not report the results of serum cryptococcal antigen, because it was not detected in our hospital. Second, we could not provide more information on the virulence factors of the infecting strain since we did not undertake the necessary identification. Third, the function of humoral or cellular immunity needs to be further clarified in patients to ensure their immune status.

\section{CONCLUSIONS}

Patients with cryptococcemia present with nonspecific symptoms. Fever, septic shock, and meningeal involvement were reported commonly among patients with cryptococcemia. AIDS, malignancies, and immunosuppressive therapy were identified as the three main predisposing conditions in our study. Patients with cryptococcemia demonstrated a high risk of mortality in the immunosuppressed group. However, early diagnosis, prompt treatment, and appropriate antifungal therapy could improve the prognosis of patients with cryptococcemia.

\section{ACKNOWLEDGEMENTS}

We thank the participants of the study with respect. We also thank Maija Eliina for critically reading this manuscript.

Funding. The collection, analysis, and interpretation of data, manuscript writing and the journal's Rapid Service Fees were supported by grants from the National Natural Sciences Foundation of China (no. 82070613), the National Natural Sciences Foundation of Hunan Province (no.2019JJ30041), and the Innovation-Driven Project of Central South University (no. 2020CX044).

Authorship. All named authors meet the International Committee of Medical Journal Editors (ICMJE) criteria for authorship for this article, take responsibility for the integrity of the work as a whole, and have given their approval for this version to be published.

Author Contributions. RC Chen and Y Huang designed the study. RC Chen, PC Zhou, $\mathrm{X}$ Zhong, MX Zou, and YM Li collected and analyzed the data. RC Chen and Yiya Zhang wrote the manuscript. All authors read and approved the final manuscript.

Prior Presentation. This manuscript is based on work that has been previously presented in the online conference of infectious disease in Changsha, Hunan province, China, on Feb 20, 2020.

Disclosures. Ruochan Chen, Yiya Zhang, Pengcheng Zhou, Xiao Zhong, Mingxiang Zou, Yanming Li and Yan Huang declare no competing non-financial/financial interests.

Compliance with Ethics Guideline. The study protocol was approved by the Ethics Committee of Xiangya Hospital (no.2018091080). Data of the patients submitted were anonymized.

Data Availability. All data generated or analyzed during this study are included in this 
published article/as supplementary information files.

Open Access. This article is licensed under a Creative Commons Attribution-NonCommercial 4.0 International License, which permits any non-commercial use, sharing, adaptation, distribution and reproduction in any medium or format, as long as you give appropriate credit to the original author(s) and the source, provide a link to the Creative Commons licence, and indicate if changes were made. The images or other third party material in this article are included in the article's Creative Commons licence, unless indicated otherwise in a credit line to the material. If material is not included in the article's Creative Commons licence and your intended use is not permitted by statutory regulation or exceeds the permitted use, you will need to obtain permission directly from the copyright holder. To view a copy of this licence, visit http://creativecommons.org/licenses/by$\mathrm{nc} / 4.0 /$.

\section{REFERENCES}

1. Chen SC, Korman TM, Slavin MA, et al. Antifungal therapy and management of complications of cryptococcosis due to Cryptococcus gattii. Clin Infect. 2013;57:543-51.

2. Yuchong C, Fubin C, Jianghan C, et al. Cryptococcosis in China (1985-2010): review of cases from Chinese database. Mycopathologia. 2012;173: 329-35.

3. Archibald LK, McDonald LC, Rheanpumikankit S, et al. Fever and human immunodeficiency virus infection as sentinels for emerging mycobacterial and fungal bloodstream infections in hospitalized patients $>/=15$ years old. Bangk J Infect Dis. 1999;180:87-92.

4. Diamond RD, Bennett JE. Prognostic factors in cryptococcal meningitis. A study in 111 cases. Ann Intern Med. 1974;80:176-81.

5. Pasqualotto AC, Bittencourt Severo C, de Mattos OF, Severo LC. Cryptococcemia. An analysis of 28 cases with emphasis on the clinical outcome and its etiologic agent. Rev Iberoam Micol. 2004;21:143-6.
6. Jean SS, Fang CT, Shau WY, et al. Cryptococcaemia: clinical features and prognostic factors. QJM. 2002;95:511-8.

7. Singer M, Deutschman CS, Seymour CW, et al. The Third International Consensus Definitions for Sepsis and Septic Shock (Sepsis-3). JAMA. 2016;315: 801-10.

8. Maertens JA, Girmenia C, Brüggemann RJ, et al. European guidelines for primary antifungal prophylaxis in adult hematology patients: summary of the updated recommendations from the European Conference on Infections in Leukaemia. J Antimicrob Chemother. 2018;73(12):3221-30.

9. Illnait-Zaragozi MT, Martinez-Machin GF, Fernandez-Andreu CM, Perurena-Lancha MR, Hagen F, Meis JF. Cryptococcus and cryptococcosis in Cuba. A minireview. Mycoses. 2014;57:707-17.

10. Chuck SL, Sande MA. Infections with Cryptococcus neoformans in the acquired immunodeficiency syndrome. N Engl J Med. 1989;321:794-9.

11. Hawkins C, Armstrong D. Fungal infections in the immunocompromised host. Clin Haematol. 1984;13:599-630.

12. Zuger A, Louie E, Holzman RS, Simberkoff MS, Rahal JJ. Cryptococcal disease in patients with the acquired immunodeficiency syndrome. Diagnostic features and outcome of treatment. Ann Intern Med. 1986;104:234-40.

13. Mabee CL, Mabee SW, Kirkpatrick RB, Koletar SL. Cirrhosis: a risk factor for cryptococcal peritonitis. Am J Gastroenterol. 1995;90:2042-5.

14. Marwaha RK, Trehan A, Jayashree K, Vasishta RK. Hypereosinophilia in disseminated cryptococcal disease. Pediatr Infect Dis J. 1995;14:1102-3.

15. Sia JK, Georgieva M, Rengarajan J. Innate immune defenses in human tuberculosis: an overview of the interactions between mycobacterium tuberculosis and innate immune cells. J Immunol Res. 2015;2015:747543.

16. Killick KE, Ní Cheallaigh C, O’Farrelly C, et al. Receptor-mediated recognition of mycobacterial pathogens. Cell Microbiol. 2013;15(9):1484-95.

17. Liu $\mathrm{CH}$, Liu $\mathrm{H}, \mathrm{Ge}$ B. Innate immunity in tuberculosis: host defense vs pathogen evasion. Cell Mol Immunol. 2017;14(12):963-75.

18. Perfect JR, Dismukes WE, Dromer F, et al. Clinical practice guidelines for the management of cryptococcal disease: 2010 update by the Infectious Diseases Society of America. Clin Infect. 2010;50: 291-322. 